\title{
La gravité de l'infection par le VIH
}

Bernard Hirschel ${ }^{a}$, Rachel Spycherb, Maximilien Catsiyannis ${ }^{c}$, Noémie Fracheboud ${ }^{\text {, }}$, Christophe Combescure ${ }^{e}$

${ }^{a}$ Prof. Dr med.; ${ }^{b}$ infirmière d'étude; ${ }^{c, d}$ étudiants en médecine; ${ }^{e}$ statisticien; tous aux Hôpitaux Universitaires de Genève

Est punissable celui qui prétend être séronégatif tandis qu'il se sait infecté, puis transmet le VIH à son partenaire lors d'un contact sexuel non protégé. Mais comment qualifier juridiquement ce délit? S'agit-il d'une lésion corporelle simple (amende ou prison jusqu'à trois ans) ou grave (jusqu'à 10 ans de prison)?

\section{Le procès}

En 2003 à Zurich, un homme que nous appellerons A, infecta son ami B par le VIH. Des années après B intenta un procès à $\mathrm{A}$, car celui-ci l'aurait trompé sur sa séropositivité et l'aurait ainsi amené à consentir à des relations sexuelles non protégées. Le tribunal de district condamna A à 33 mois de prison, dont 6 fermes, sur la base de l'article 122 du code pénal suisse. S'y ajoutent des frais de justice, une indemnisation et une réparation pour tort moral, au total environ 100000 francs [1].

A fit appel au tribunal cantonal (qui confirma le jugement), puis au tribunal fédéral. En 2013, celui-ci demanda au tribunal cantonal de Zurich de commanditer une expertise sur la gravité de l'infection VIH en 2003 et en 2013; ensuite, basé sur cette expertise, de trancher entre l'application de l'article 122 et 123 [2].

Art. 122 du code pénal suisse

Celui qui, intentionnellement, aura blessé une personne de façon à mettre sa vie en danger, celui qui, intentionnellement, aura mutilé le corps d'une personne, un de ses membres ou un de ses organes importants ou causé à une personne une incapacité de travail, une infirmité ou une maladie mentale permanentes, ou aura défiguré une personne d'une façon grave et permanente, celui qui, intentionnellement, aura fait subir à une personne toute autre atteinte grave à l'intégrité corporelle ou à la santé physique ou mentale, sera puni d'une peine privative de liberté de dix ans au plus ou d'une peine pécuniaire de 180 jours-amende au moins.

Art. 123

Celui qui, intentionnellement, aura fait subir à une personne une autre atteinte à l'intégrité corporelle ou à la santé sera, sur plainte, puni d'une peine privative de liberté de trois ans au plus ou d'une peine pécuniaire.

\section{La gravité de l'infection par le VIH}

\section{L'effet du traitement antirétroviral hautement actif (HAART)}

Sans traitement efficace la mortalité atteint $50 \% 12$ ans après l'infection par le VIH [3]. L'histoire naturelle de la maladie a été modifiée radicalement depuis 1996, année de l'introduction des trithérapies hautement actives (HAART). La figure montre l'incidence d'infections opportunistes ou de tumeurs classant Sida avant et après le début de HAART dans l'étude suisse de cohorte [4].

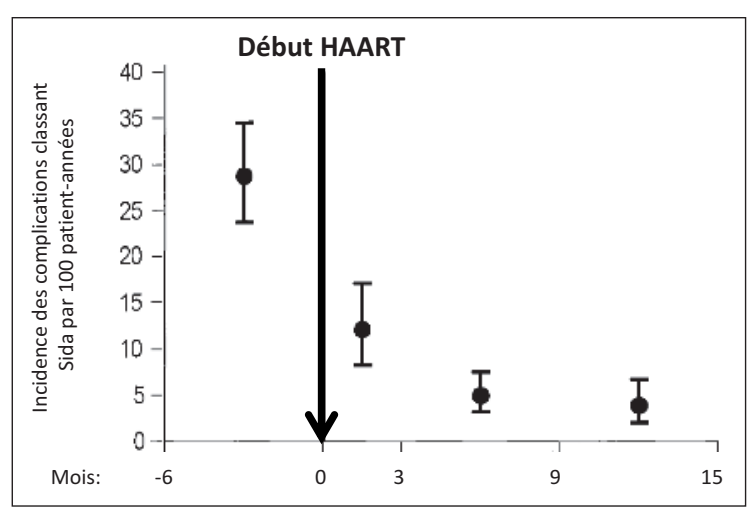

Abbildung 1: Incidence du Sida avant et après le début du traitement anti-rétroviral hautement actif dans l'étude suisse de cohorte VIH.

Cependant, HAART n'était pas un traitement simple. En 1996, la combinaison d'indinavir, zidovudine et lamivudine dominait. Les patients prenaient 2 comprimés d'indinavir à 6 heures du matin, l'estomac vide, puis un comprimé de zidovudine et un comprimé de lamivudine avec le petit déjeuner, à 14 heures de nouveau de l'indinavir, zidovudine, et lamivudine au souper, et enfin de l'indinavir à 22 heures [5]. Des effets secondaires diminuaient la qualité de vie: diarrhées, 
nausées, anémie, calculs rénaux, ongles incarnés, ainsi que la lipodystrophie, une modification de l'apparence corporelle avec une perte du tissu adipeux sous-cutanée au visage et aux membres, couplée parfois à une accumulation de la graisse abdominale.

\section{Progrès additionnels de 1996 à 2015}

Des médicaments plus récents, par exemple efavirenz, tenofovir et darunavir, ne demandent qu'une prise journalière. Les effets secondaires, en particulier la lipodystrophie, sont devenus plus rares, facilitant le respect des prescriptions thérapeutiques, et par là le succès du traitement $[6,7]$.

Un autre problème fut largement maîtrisé: la résistance du virus au traitement. Etravirine, raltegravir et darunavir, utilisés en combinaison, se sont avérés efficaces même en cas de résistances multiples. Les patients non traitables ont presque disparu de nos consultations [8].

Plusieurs substances actives sont maintenant combinées en une seule pilule. Les médicaments Atripla ${ }^{\circledR}$, Eviplera ${ }^{\circledR}$, Stribild ${ }^{\circledR}$ ou Triumeq ${ }^{\circledR}$ permettent un traitement efficace et bien toléré avec une seule pilule par jour.

\section{Survivre avec le VIH, hier et aujourd'hui}

S'il s'agit d'évaluer la gravité de l'infection par le VIH, la question de l'espérance de vie joue un rôle important. Il a déjà été mentionné que sans traitement, la mortalité atteint $50 \%$ après 12 ans de séropositivité. Vu l'âge moyen de 30 ans au moment de l'infection, cela représente une diminution drastique de l'espérance de vie. Mais qu'en est-il aujourd'hui, après prise en compte de l'effet du traitement? La réponse à cette question n'est pas simple:
- Le temps d'observation et encore court. Une courbe de survie est complète seulement quand tous les sujets sont décédés. La population des séropositifs étant relativement jeune, il faudrait attendre plus de 50 ans!

- Les médicaments efficaces n'ont que 20 ans. Des effets secondaires ou une perte d'efficacité tardifs sont improbables; dans l'hypothèse qu'ils existeraient, ils pourraient influencer la survie sans qu'on puisse les détecter aujourd'hui.

- Les traitements continuent à s'améliorer. Ceci est cependant difficile à démontrer, car le suivi est trop bref pour ceux qui ont débuté le traitement récemment.

- Même si le temps d'observation est limité, il est possible de comparer la survie des séropositifs avec un groupe contrôle VIH-négatif, par exemple la population suisse du même âge. Si la mortalité est similaire, on s'attend, par extrapolation, à une espérance de vie également semblable. A cet égard, il faut considérer que les séropositifs et les séronégatifs ne se distinguent pas seulement quant au VIH. Par exemple, il y a plus de fumeurs parmi les séropositifs, ce qui diminue leur espérance de vie. Beaucoup sont co-infectés par les virus de l'hépatite, causes d'insuffisance hépatique et de cancers du foie parfois mortels. En 2009, seulement $9 \%$ des décès des séropositifs en Suisse étaient dus au Sida [9]!

\section{Le pronostic de l'infection VIH, données de l'étude suisse de cohorte (SHCS)}

Un travail paru en 2003 a examiné la mortalité dans la SHCS entre 1997 et 2001. La population séropositive fut divisée en plusieurs catégories et la mortalité fut comparée à celle de la population suisse appariée sur l'âge

Tableau 1: Mortalité des séropositifs sous HAART efficace, 1997-2001.

\begin{tabular}{llll}
\hline Catégorie & $\begin{array}{l}\text { Mortalité des patients VIH par } \\
\mathbf{1 0 0 0} \text { années d'observation }\end{array}$ & $\begin{array}{l}\text { Valeur de référence: } \\
\text { Mortalité de la population suisse } \\
\text { comparable }\end{array}$ & Surmortalité \\
\hline $\mathrm{HCV}-, \mathrm{VIH}+$ & 7,8 & 3,6 & 4,2 \\
\hline $\mathrm{HCV}+, \mathrm{VIH}+$ & 23,4 & $1,7^{*}$ & 21,7 \\
\hline
\end{tabular}

* La co-infection par l'hépatite $\mathrm{C}$ et le VIH est particulièrement fréquente chez les utilisateurs de drogues, qui sont en moyenne plus jeunes que les HIV-monoinfectés, d'où la valeur de référence inférieure.

Tableau 2: Mortalité des séropositifs sans HAART ou sous HAART inefficace, 1997-2001.

\begin{tabular}{|c|c|c|c|}
\hline Catégorie & $\begin{array}{l}\text { Mortalité des patients VIH par } 1000 \\
\text { années d'observation }\end{array}$ & Valeur de référence & Surmortalité \\
\hline HCV-, VIH+ & 123 & 5,6 & 117,4 \\
\hline $\mathrm{HCV}_{+}, \mathrm{VIH}+$ & 115 & 2,3 & 112,7 \\
\hline
\end{tabular}


Tableau 3: Questionnaire de l'enquête.

Comparez, s.v.p., les maladies suivantes avec I'infection VIH (séropositivité).

1. Avoir une tension artérielle élevée, c'est
$\square \quad$ Plus grave
$\square$ A peu près aussi grave
$\square \quad$ Moins grave...que d'être séropsitif(-ve) en 2014

2. Devenir paralysé des jambes et devoir se déplacer en fauteuil roulant, c'est

$\square$ Plus grave $\quad \square$ A peu près aussi grave $\quad \square \quad$ Moins grave...que d'être séropsitif(-ve) en 2014

3. Avoir une arthrose et devoir se déplacer avec une canne, c'est

$\square$ Plus grave $\quad \square$ A peu près aussi grave $\quad \square \quad$ Moins grave...que d'être séropsitif(-ve) en 2014

4. Avoir un diabète traité avec pilules c'est

$\square$ Plus grave $\quad \square$ A peu près aussi grave $\quad \square \quad$ Moins grave...que d'être séropsitif(-ve) en 2014

5. Avoir un diabète traité avec régime, pilules et injections journalières, c'est

$\square$ Plus grave $\quad \square$ A peu près aussi grave $\quad \square \quad$ Moins grave...que d'être séropsitif(-ve) en 2014

6. Perdre la vue d'un œil, c'est

$\square$ Plus grave $\quad \square$ A peu près aussi grave $\quad \square \quad$ Moins grave...que d'être séropsitif(-ve) en 2014

7. Devenir aveugle, c'est

$\square$ Plus grave $\quad \square$ A peu près aussi grave $\quad \square \quad$ Moins grave...que d'être séropsitif(-ve) en 2014

8. Recevoir un coup qui laisse une vilaine cicatrice à travers le visage, c'est

$\square$ Plus grave $\quad \square$ A peu près aussi grave $\quad \square \quad$ Moins grave...que d'être séropsitif(-ve) en 2014

9. Avoir un cancer qui doit être opéré (comme par exemple un cancer du sein chez la femme, ou un cancer prostatique chez I'homme, c'est

$\square$ Plus grave $\quad \square$ A peu près aussi grave $\quad \square \quad$ Moins grave...que d'être séropsitif(-ve) en 2014

10. Avoir un cancer sans espoir de guérison, c'est

$\square$ Plus grave $\quad \square$ A peu près aussi grave $\quad \square \quad$ Moins grave...que d'être séropsitif(-ve) en 2014

(population de référence). Les données démontrent l'effet de HAART qui diminue la mortalité de plus de 90\% (de 123 par 1000 patient-années à 7,8, voir la comparaison du tableau 2 avec le tableau 1). D'autre part, chez ceux qui sont traités par HAART, le tableau 1 démontre l'influence prépondérante de la co-infection par le virus de l'hépatite $C$ sur la mortalité.

Depuis la parution de cet article, la mortalité a continué de baisser [11]; un début de traitement après 2001 est associé à un meilleur pronostic qu'un début entre 1996 et 2000 (référence [11], tableau 2). Mais une différence subsiste entre la mortalité des séropositifs et celle des personnes souscrivant une assurance vie (qui est inférieure à la mortalité de la population générale).

\section{Qualité de vie, effets secondaires des médica-} ments, vieillissement précoce, procréation

HAART, c'est la prise de plusieurs médicaments, à vie. Un suivi médical régulier, tous les trois à six mois, reste nécessaire. De nombreux patients seraient d'accord de prendre des risques considérables si en échange ils pouvaient se débarrasser définitivement du VIH; espoir irréaliste à l'heure actuelle.

Les effets secondaires ne pouvaient pas être évités au début de HAART. La situation s'est améliorée depuis; on a maintenant le choix entre plus de 20 médicaments qui permettent de trouver une combinaison bien tolérée dans presque tous les cas. Il existe des craintes quant à la promotion de maladies cardio- vasculaires qui en combinaison avec l'infection VIH elle-même accélèreraient le processus de vieillissement; ce qui n'est pas avéré à l'heure actuelle.

La stigmatisation ou au moins la crainte de celle-ci, pèse lourdement sur de nombreux séropositifs. Elle a des conséquences pratiques, par exemple la difficulté à souscrire une assurance vie, ce qui diminue la solvabilité. Certains n'osent pas changer de poste de travail, car ils redoutent que leur séropositivité soit connue dans l'entreprise par l'intermédiaire de la caisse de pension. Les craintes quant à la contagiosité par voie sexuelle sont objectivement infondées, car la transmission à partir d'un patient traité et dont la virémie est indétectable, est pratiquement impossible. De même, la transmission de la mère vers le nouveau-né a disparu. Malgré tout, la communication entre partenaires sexuels reste problématique. Nombreux sont ceux qui rapportent avoir été délaissés à cause de leur séropositivité, tandis que d'autres n'osent même pas envisager une nouvelle relation.

\section{Ce qu'en pensent les gens - une enquête}

Dans un état démocratique, l'appréciation d'un délit reflète, au moins partiellement, l'opinion publique. D'où l'intérêt de savoir ce qu'en pense le citoyen: VIH, lésion corporelle simple ou grave? Quel est le reflet des progrès thérapeutiques dans les opinions d'un public plus large? 
Tableau 4: Résultat de I'enquête.

\begin{tabular}{|c|c|c|c|}
\hline Population interrogée & $\mathbf{N}$ & Score & $\mathbf{P}$ (comparaison du score) \\
\hline Etudiants en médecine & 217 & 6,9 & $\begin{array}{l}\text { - }<10^{-3} \text { (test de } t \text { ) entre étudiants en médecine } \\
\text { et droit } \\
\text { - Non significatif entre étudiants en médecine } \\
\text { et employés d'hôpital }\end{array}$ \\
\hline Toutes personnes VIH-négatives & 737 & 7,2 & \multirow{2}{*}{$\bullet<10^{-3}$ (test de t) entre $\mathrm{VIH}$-positifs et négatifs } \\
\hline VIH-positifs & 297 & 4,8 & \\
\hline
\end{tabular}

Afin d'obtenir une réponse à cette question, nous avons organisé, en 2014, une enquête dont le but était la comparaison de l'infection VIH à d'autres maladies (voir tableau 3).

Ce questionnaire fut rempli par 1043 personnes, dont 297 séropositifs à Genève et à Annecy. 737 étaient VIH-négatifs, environ 200 étudiants en médecine et 200 étudiants en droit, 236 employés à l'hôpital universitaire de Genève (surtout des médecins et des infirmières), ainsi qu'une centaine d'autres.

L'évaluation des réponses repose sur l'idée que voici:

- Qui considère que le VIH est "grave», classera de nombreuses maladies comme étant moins grave ou aussi grave que le VIH

- Au contraire celui qui pense que le VIH est anodin: pour lui, il n'existe guère de maladies moins graves. En conséquence, chaque questionnaire rempli a reçu un score correspondant au nombre de réponses cochées «moins graves» ou «à peu près aussi grave». Les résultats sont résumés dans le tableau 4. Plus le score est élevé, plus le VIH est considéré comme grave comparativement aux autres pathologies citées dans le questionnaire.

Les réponses sont différentes selon la population questionnée. Les VIH+ trouvent que l'infection VIH est à peu près aussi grave qu'une arthrose ou un diabète, tandis que les séronégatifs la trouvent comparable à une apoplexie avec hémiplégie. La figure 2 illustre clairement le contraste entre les deux groupes.

Quelle est l'origine de ses différences? Deux explications nous apparaissent plausibles:

- Les nouveaux traitements ont bouleversé le pronostic de l'infection par le VIH. Les patients vivent ce progrès dans leur propre corps, tandis que les étudiants en médecine et autres professionnels de la santé le connaissent au moins partiellement par leur enseignement ou leurs contacts avec les patients. L'image du VIH que se fait un publique plus

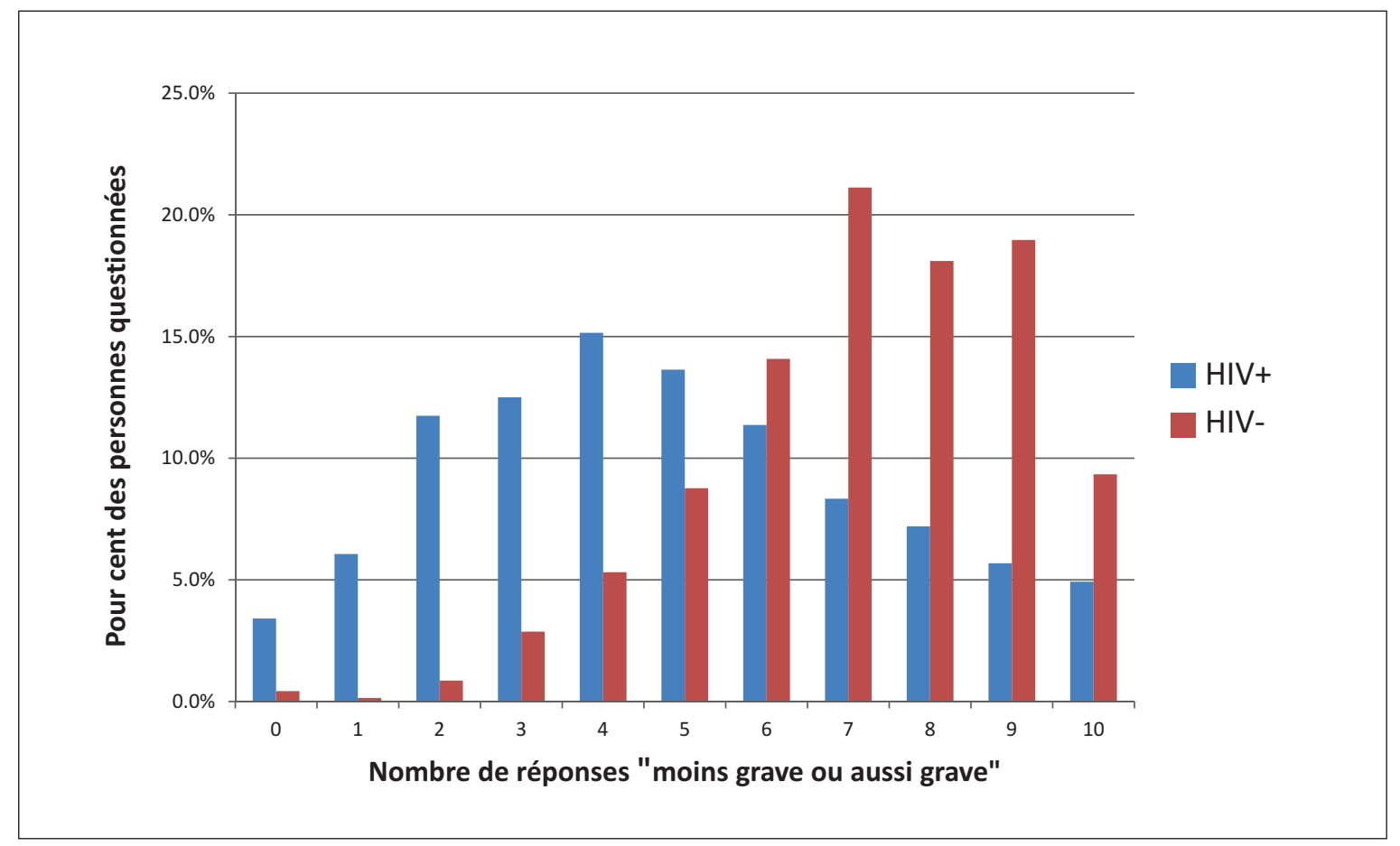

Figure 2: Comparaison des réponses des séropositifs et séronégatifs. 
large a au contraire du retard, expliquant ainsi la différence entre étudiants en médecine et en droit.

- Les personnes souffrant de maladies ou de blessures les considèrent comme moins graves que les non-malades et non-blessés, selon le principe: «on s'habitue à tout» [12].

\section{La décision du tribunal}

Comment auriez-vous décidé, cher lecteur, chère lectrice? Faut-il juger A selon l'article 122 (lésion corporelle grave) ou 123 (lésion corporelle simple)?

La deuxième cour pénale du tribunal cantonal de $\mathrm{Zu}$ rich a pris sa décision le 29 juin 2015 [13]. En tenant compte de l'expertise, elle a confirmé qu'il s'agissait d'une lésion corporelle grave.

La cour a cependant révisé la peine. Plus de 10 ans se sont écoulés depuis le délit sans que l'accusé ait commis un autre crime; il avait même commencé à travailler pour la prévention du VIH. La durée de la peine fut diminuée à 24 mois, ce qui permet le sursis, et la compensation pour tort moral fut réduite de 50000 .- à 35000.-

L'accusé a de nouveau fait appel au tribunal fédéral - il y aura donc une suite. Il est peu probable que l'instance suprême casse le jugement, puisqu'au printemps 2015, elle a confirmé que l'infection par le VIH correspondait à une lésion corporelle grave - dans une histoire fort différente, celle du «guérisseur de Berne»[14].

Correspondance:

Pr B. Hirschel

13 , chemin des Lucioles

CH-1234 Vessy

Tél. 0227842695

bernard.hirschel[at]

hcvge.ch

\section{Remerciements}

Mme Susanne Polydor a revu les aspects juridiques. Les docteurs J.-P. Bru et Jacques Gillet ont interrogé les patients VIH à Annecy et le Pr S. Marchand les étudiants en droit à Genève. Qu'ils soient ici chaleureusement remerciés.

\section{Références}

$1 \mathrm{http} / / /$ www.gerichte-zh.ch/fileadmin/user_upload/entscheide/ oeffentlich/SB110505-O1.pdf

2 http://www.bger.ch/index/juridiction/jurisdiction-inherit-template/jurisdiction-recht/jurisdiction-recht-leitentscheide1954. htm, Urteil 139 IV 214

3 Koblin BA, van Benthem BH, Buchbinder SP, Ren L, Vittinghoff E, Stevens CE et al.: Long-Term Survival after Infection with Human Immunodeficiency Virus Type1 (HIV-1) among Homosexual Men in Hepatitis B Vaccine Trial Cohorts in Amsterdam, New York City, and San Francisco, 1978-1995. American Journal of Epidemiology 1999, 1999; 150:1026-30.

4 Ledergerber B, Egger M, Erard V, Weber R, Hirschel B, Furrer H, et al. AIDS-Related opportunistic illnesses occurring after initiation of patent antiretroviral therapy. The Swiss HIV Cohort Study. JAMA 1999; 282(23):2220-6

5 Hammer SM, Squires KE, Hughes MD, Grimes JM, Demeter LM, Currier JS et al. A controlled trial of two nucleoside analogues plus indinavir in persons with human immunodeficiency virus infection and CD4 cell counts of 200 per cubic millimeter or less. N Engl J Med. 1997 337(11):725-33.

6 Staszewski S, Morales-Ramirez J, Tashima KT, Rachlis A, Skiest D, Stanford J et al. Efavirenz plus zidovudine and lamivudine, efavirenz plus indinavir, and indinavir plus zidovudine and lamivudine in the treatment of HIV-1 infection in adults. Study 006 Team. N Engl J Med. 1999 Dec 16;341(25):1865-73.

7 Sax PE, Tierney C, Collier AC, Fischl MA, Mollan K, Peeples L et al. Abacavir-lamivudine versus tenofovir-emtricitabine for initial HIV-1 therapy. N Engl J Med. 2009;361 (23):2230-40

8 Fagard C, Colin C, Charpentier C, Rami A, Jacomet C, Yeni P et al. Long-term efficacy and safety of raltegravir, etravirine, and darunavir/ritonavir in treatment-experienced patients: week 96 results from the ANRS 139 TRIO trial. J Acquir Immune Defie Syndr. 2012;59(5):489-93

9 Weber R, Ruppik M, Rickenbach M, Spoerri A, Furrer H, Battegay M et al. Decreasing mortality and changing patterns of causes of death in the Swiss HIV Cohort Study. HIV Medicine 2013;14:195

10 Jaggy C1, von Overbeck J, Ledergerber B, Schwarz C, Egger M, Rickenbach M et al. Mortality in the Swiss HIV Cohort Study (SHCS) and in the Swiss general population. Lancet 2003; 362:877-9.

11 Kaulich-Bartz J,Dam W, May MT, Ledergerber B, Widmer U, Phillips AN, Grabar S et al. Insurability of HIV-positive people treated with antiretroviral therapy inEurope: collaborative analysis of HIV cohort studies. AlOS 2013; 27:1641-55

12 Kahnemann D. Thinking fast and slow. Penguin Books, Penguin Random House UK, 2012, p 404-6.

13 Obergericht des Kantons Zürich, II. Strafkammer, Urteil vom 29.6.2015, SB130132

$14 \mathrm{http} / /$ www.bger.ch/index/juridiction/jurisdiction-inherittemplate/jurisdiction-recht/jurisdiction-recht-leitentscheide1954 htm, BGE 141 IV 97 